\title{
Payoff Externality and Performance-Based Contracts for Transport Infrastructure
}

\author{
Lei Shi ${ }^{1}$, Masamitsu Onishi ${ }^{2}$ and Kiyoshi Kobayashi ${ }^{3}$ \\ ${ }^{1}$ Dalian University of Technology \\ ${ }^{2,3}$ Kyoto University \\ ${ }^{1}$ leishi@dlut.edu.cn, ${ }^{2}$ onishi.masamitsu.7e@kyoto-u.ac.jp, \\ ${ }^{3}$ kobayashi.kiyoshi.6n@kyoto-u.ac.jp
}

\begin{abstract}
This paper examines the adverse selection and moral hazard issues of the performance-based contracts in transport infrastructure projects, which are caused by the pay-off externality between the contracts signed out in the different times and identified by the private agent monitoring. The adverse selection and moral hazard cannot be deterred by the penalty systems and competitive tendering, if the operation is carried out by a sequence of the individual short-term contracts. On the other hand, the adverse selection and moral hazard can be efficiently deterred by the long-term contracts with a single agent internalizing the pay-off externality, as far as the agent is bind to the contract throughout the contract periods. However, the efficiency of the long-term contracts is flawed if the breach of the contract is made by the agent. The paper also investigates the means to deter the strategic breaches of the agent.
\end{abstract}

Keywords: moral hazard, performance-based contract, adverse selection, contract efficiency

\section{Introduction}

The performance-based contracts (hereafter performance-based contracts 0 ) have been adopted for the management and operation of transport infrastructure. The performancebased contracts specify the required performance of the infrastructure and the payments based on how well the agent complies with the required performance but do not undertake the payments based on the amount of works executed.

The performance standard of the infrastructure involves quantitatively describable factors (hereafter describable performance factors) such as wheel track overcharges and road pavement rages, and quantitatively indescribable factors (hereafter indescribable performance factors) such as various damages and performance degradations. The former factors which are observable and verifiable can be specified in the performance-based contracts because these factors can be defined as the required performance by means of objective index. However the latter factors cannot be described in the contracts since they are observable but unverifiable. In this sense, the performance-based contracts must be incomplete contracts [1][2].

The performance-based contract is supposed to be repeated by multiple short-term contracts. There is an extensive literature analyzing efficiency issues in multi-period contracts. Baron and Besanko [3] assume a case in which principal can commit to a 2period contract (long-term contract). They show that the optimal long-term contract is structured by repeating the optimal one-period contract. On the contrary, Freixas, Guesnerie and Tirole [4], Laffont and Tirole [5][6] analyze the optimal policy in a case where principal cannot commit himself to a revision procedure, whereby the main focus is upon the ratchet effect which refers to a situation where the principal revises the latter period contract by taking into account the additional information he obtained in the 
previous period. These studies assume that the principal does not have any information about the agent's type at the beginning of the first period, i.e., the adverse selection problem occurs. On the other hand, Rogerson [7] deals with the moral hazard problem, where the only private information is the agent's choice of actions. Rogerson [7] indicates the optimal long-term contracts will be efficient afterwards by assuming that the agent has no way to access to a credit market. In addition, Fudenberg, Holmström and Milgrom [8], Chiappori, Macho, Rey and Salanié [9] analyze the effects of agent access to the credit market in terms of the optimal long-term contract efficiencies. The conclusion of these studies is that sequential efficiency will fail if the agent can borrow and save as he likes. This result suggests that the optimal public contracts should be short-term contracts, since the private companies attending the public project have access to the credit market undoubtedly. However, many current performance-based contracts are long-term contracts so it is difficult to explain within the theoretic framework mentioned above.

In this paper, the performance-based contracts are classified into two groups; shortterm and long-term contracts. We define a life period as the shortest unit of time during which the asset is renewed. The short-term contract divides the life period in two contractual terms, and the performance-based contract is signed during each contractual term. On the other hand, the long-term contract refers to the performance-based contract concluded in the whole life period. In the short term contract, the outcomes of the maintenance and operation during the first contractual term will influence the cost coming about during the second term, i.e., the dynamic economic externality (hereafter payoff externality) arises. However, there are a few studies that analyzed payoff externality. Schmitz [10] compared the integration contract with the separation contract by assuming that the outcome of the first period has a positive externality effect upon the agent's effort in the second period. Under integration, the agent might be tempted to evade in the first period in order to increase the rent in the second period. In contrast to the Schmitz's study, this paper focuses that the outcome of the first period has a negative externality effect upon the costs coming about in the first period.

In the performance-based contract, the agent might implement private monitoring on the performance before the government's public monitoring in order to match the performance to the required performance in advance. Following the game theory framework, this paper defines the public monitoring as an action to obtain the signals that are observable to all players. However, the government cannot identify whether the performance satisfies the requirement without implementing the monitoring. On the other hand, the private company is capable to catch some private information about the real performance through the diary of maintenance and operational works. In this way, the private monitoring refers to the information catch which can be observed only by a particular player [11]. If the private monitoring is done before the public monitoring, the monitoring implemented by the government is public while the monitoring implemented by the private company is private.

The describable performance factors might be related to the indescribable performance factors. Now consider the agent's action under the short-term contract. If the agent exerts preventative recovery, not only the describable performance factors match the required standards, but also the level of the indescribable performance factors doesn't decrease. However, the recovery costs might increase in the second period (payoff externality) and the indescribable performance factors lessen if the agent evades. In the situation that only the public monitoring information is available, the agent can be obligated to carry on the preventative recovery by the established penalty clauses. However, the agent should find the real performance failures through private monitoring and recover the performance to fulfill the required level before the public monitoring not to pay the penalty fee. It means that the describable performance factors are recovered but the payoff externality arises from the neglect of the preventative recovery, i.e., the moral hazard occurs. The incremental costs due to the payoff externality are borne by the agent who is responsible 


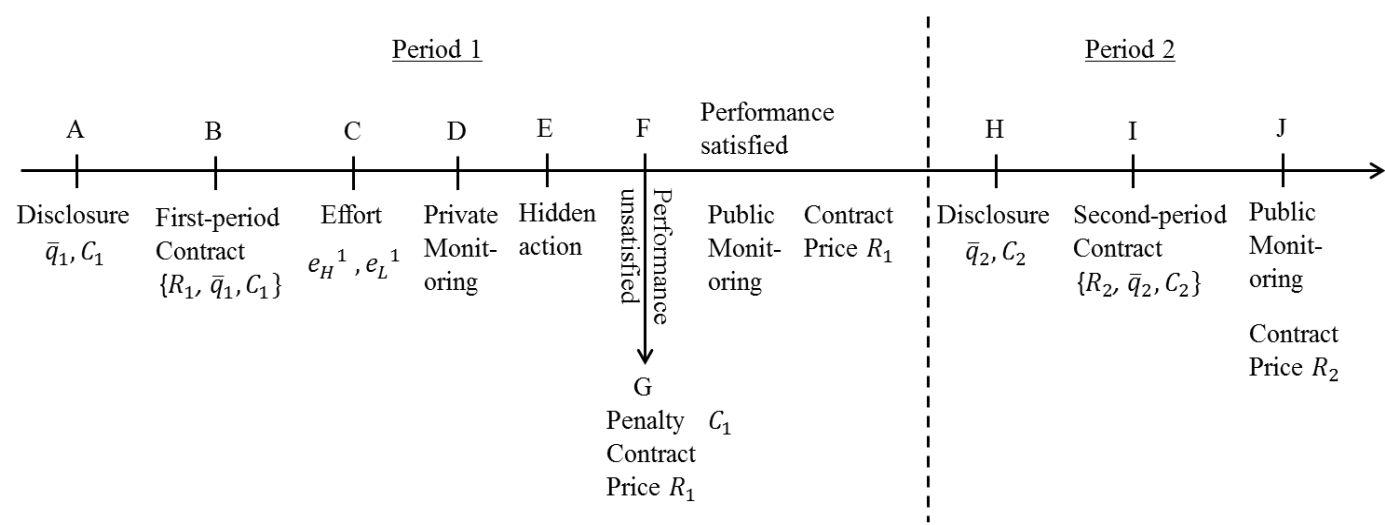

Figure 1. Timing of the Short-Term Contract

for the asset performance during the second period. As a result, the agent who neglects the preventative recovery should win the contract at low price at the beginning of the first period, i.e., the adverse selection occurs.

Under long-term contract, the main issue is whether the agent commits to complete the contract. If the agent commits to complete the contract, the long-term contract is desirable since the payoff externality is internalized [12][13]. However, both the moral hazard and adverse selection might arise if the agent with limited liability breaches the contract (falls into bankruptcy) intentionally. The uncertainty of payoff externality is assumed, the agent will avoid the additional cost due to the payoff externality, i.e., the moral hazard arises. Additionally, the adverse selection occurs in a low-priced tender for the long-term contract. In this sense, an institutional framework for deterring the strategic breaches of the agent is necessary under long-term contract.

The remainder of the paper is organized as follows. Section 2 and 3 formalize the short-term and long-term contracts as incomplete contracts, respectively. Section 4 investigates the intentional cancellation problem of the long-term contracts. Concluding remarks follow in Section 5.

\section{Short-term Contracts}

\subsection{Assumptions}

The short-term contract is interpreted as a 2-period game as shown in Figure 1. At the beginning of period 1 , the government discloses the required performance $\bar{q}_{1}$ and the penalty fee $C_{1}$. Then a tender for determining the contract price is implemented (point A). The government closes the short-term contract with the winning bidder (point $\mathrm{B}$ ). The contract price $R_{1}$, the required performance $\bar{q}_{1}$ and the penalty fee $C_{1}$ are described in the first-period contract. The agent then chooses a level of effort $e^{1}=\left\{e_{H}^{1}, e_{L}^{1}\right\}$ (point $\mathrm{C}$ ). Effort refers to the action of exerting preventative recovery. After that, the agent implements the private monitoring for capturing the information about the real performance and the payoff externality (point D). If the real performance fails to satisfy the required standards, the agent will recover the describable performance factors (hidden action) (point E). The government performs the public monitoring (point $F$ ). The agent pays the penalty fee $C_{1}$ if the performance doesn't meet the required standard. The raised penalty fee is fund for recovering the defective performance (point $\mathrm{G}$ ). The government pays the contract fee $R_{1}$ to the agent. The second-period contract repeats the same events that take place in the first-period. However, we only consider least possible number of events in the second-period contract in order to make the prospect of the argument clear. At the beginning of the second-period, a tender is implemented (point $\mathrm{H}$ ). The second- 
period contract specifies the contract price $R_{2}$, the required performance $\bar{q}_{2}$ and the penalty fee $C_{2}$ (point I). The contract price $R_{2}$ is paid to the agent at the end of the second-period (point $\mathrm{J}$ ). We firstly assume the contract prices as exogenous variables. The mechanism in which the tender determines the contract prices is investigated in 2.4. It is supposed that the interest rate (discount rate) is zero, and all the players are risk neutral.

\subsection{Payoff Structure}

Asymmetric information about the agent's type and the level of effort exists between the government and the agent. The former refers to hidden attribute, which bring about the adverse selection. The latter refers to hidden action, which triggers the occurrence of the moral hazard. The agent can be either efficient (hereafter good agent) $\theta_{g}^{k}$ or inefficient (hereafter bad agent) $\theta_{b}^{k}$ with respective probability $\alpha$ and $1-\alpha$. Suppose that the good agent possesses the technology for preventative recovery whereas the bad agent has no ability for preventative recovery. However the good agent might be negligent in the preventative recovery, which causes that both the describable and indescribable performance factors decrease. Thus the payoff externality due to the agent's neglect increases the maintenance and operation cost occurs in the second-period. Assume that the externality is uncertain. The uncertainty of the payoff externality is not essential under the short-term contract but it performs an important role on the breach of the long-term contract.

At the beginning of each period, the agent who is responsible for the maintenance and operational works is selected. The government has no idea about the agent's type. Denote the private information space as $\Theta=\left\{\theta_{g}^{k}, \theta_{b}^{k}\right\}$. The good agent chooses a level of effort $e^{1}=\left\{e_{H}^{1}, e_{L}^{1}\right\} . e_{H}^{1}$ represents the preventative recovery is implemented, whereas $e_{L}^{1}$ represents no preventative recovery is implemented. On the other hand, the bad agent only chooses $e_{L}^{1}$, since he possesses no technology for preventative recovery. The cost related to effort $F$ is defined as

$$
F= \begin{cases}\phi>0 & \text { if } e^{1}=e_{H}^{1} \\ 0 & \text { if } e^{1}=e_{L}^{1}\end{cases}
$$

Suppose agent's effort can partly impede the fall in the process of the asset. The real performance at the end of the first-period is denoted by $\hat{q}_{1}$. The real performance $\hat{q}_{1}$ matches the required performance $\bar{q}_{1}$ with probability $P\left(\hat{q}_{1} \geq \bar{q}_{1} \mid \theta_{i}^{1}, e_{j}^{1}\right)(i=g, b)(j=H, L)$, which depends on both the agent's type and effort. Define

$$
\left.\begin{array}{l}
P\left(\hat{q}_{1} \geq \bar{q}_{1} \mid \theta_{g}^{1}, e_{H}^{1}\right)=1 \\
P\left(\hat{q}_{1} \geq \bar{q}_{1} \mid \theta_{g}^{1}, e_{L}^{1}\right)=0 \\
P\left(\hat{q}_{1} \geq \bar{q}_{1} \mid \theta_{b}^{1}, e_{L}^{1}\right)=0
\end{array}\right\}
$$

Then the government monitors the real performance. If the result of the monitoring shows that $\hat{q}_{1}<\bar{q}_{1}$, the penalty fee $C_{1}$ will be paid by the agent.

Now suppose $C_{1}$ is larger than the ex-post recovery cost. The agent who chooses effort $e_{L}^{1}$ performs the private monitoring before the public monitoring. The agent recovers the describable performance factors, which don't meet the hidden required performance. As a result, the government cannot identify whether the agent adopts effort $e_{H}^{1}$ or not. On the contrary, the agent has no incentive to maintain the performance level into the required standards if $C_{1}$ is smaller than the ex-post recovery cost. In this sense, suppose the penalty fee is equal to the ex-post recovery cost. After confirming the penalty fee, the government pays the contract price $R_{1}$ to the agent. 
Table 1. Payoff Externality and Cost Structure

\begin{tabular}{|c|c|c|c|c|}
\hline \multirow{2}{*}{ Type of agent } & Effort & $\begin{array}{l}\text { Cost at the } \\
\text { first-period }\end{array}$ & Probability & $\begin{array}{l}\text { Cost at the } \\
\text { second-period }\end{array}$ \\
\hline \multirow{2}{*}{$\theta_{g}^{1}$} & $e_{L}^{1}$ & $C_{1}$ & $p$ & 0 \\
\cline { 2 - 5 } & $e_{H}^{1}$ & $\phi$ & $1-p$ & $M$ \\
\hline \multirow{2}{*}{$\theta_{b}^{1}$} & $e_{L}^{1}$ & $C_{1}$ & 1 & 0 \\
\cline { 4 - 5 } & & & $1-p$ & 0 \\
\hline
\end{tabular}

At the beginning of the second-period, the government enters a new contract with a new agent. The new contract specifies the required performance $\hat{q}_{2}$, contract price $R_{2}$ and the penalty fee $C_{2}$ in the second-period. In this paper it is supposed that no distinction exists between the new agent and the first-period agent if they are the parts of the same type. The government might continue the contract with the existing agent if it is found that the existing agent is a good one. However, the identification of agent's type is difficult since it depends on the indescribable performance factors which are unverifiable. In this sense, the competitive tender in the selection of a new agent is carried out at the beginning of the second period. The new contract turns out to be a short-term contract, and the moral hazard and adverse selection might also arise in the first-period contract. In the same way, the cost structure in the second-period contract is to the same as that in the first-period contract except for the payoff externality. For the sake of simplicity, we only focus on the payoff externality problem.

As the asset is renewed after the second-period, the payoff externality due to the level of effort exerted in the second-period doesn't exist. Thus, the agent only carries on the ex-post recovery without implementing the preventative recovery (the level of effort $e_{L}^{1}$ is chosen). As a result, neither the moral hazard problem nor adverse selection problem arises in the second-period. In this sense, the secondperiod contract only focuses on the payoff externality that contributes in the firstperiod contract. Suppose the normal maintenance and operation cost that arises in the second-period is zero so the additional cost due to the payoff externality is denoted by $K$, which depends on whether the first-period agent implements the preventative recovery. Define

$$
K= \begin{cases}0 & \text { (if } \left.e^{1}=e_{H}^{1}\right) \\ 0(\text { with probability } p) & \left(\text { if } e^{1}=e_{L}^{1}\right) \\ M(\text { with probability } 1-p) & \end{cases}
$$

The additional cost is observable by both of the government and new agent. According to (2), the second-period maintenance and operation cost is zero if the first-period agent exerts $e_{H}^{1}$, whereas the second-period cost is 0 or $M$ with probability $p$ or $1-p$ respectively, if the first-period agent exerts $e_{L}^{1}$. The additional cost is determined at the end of period 1. Thus, the second-period agent takes into account the contract price $R_{2}$ by using the information about the additional cost. The maintenance and operation cost structure is summarized in Table 1. Suppose the penalty fee $C_{2}$ is equal to the additional cost $M$. The government finally pays the contract price to the agent after implementing the public monitoring. 


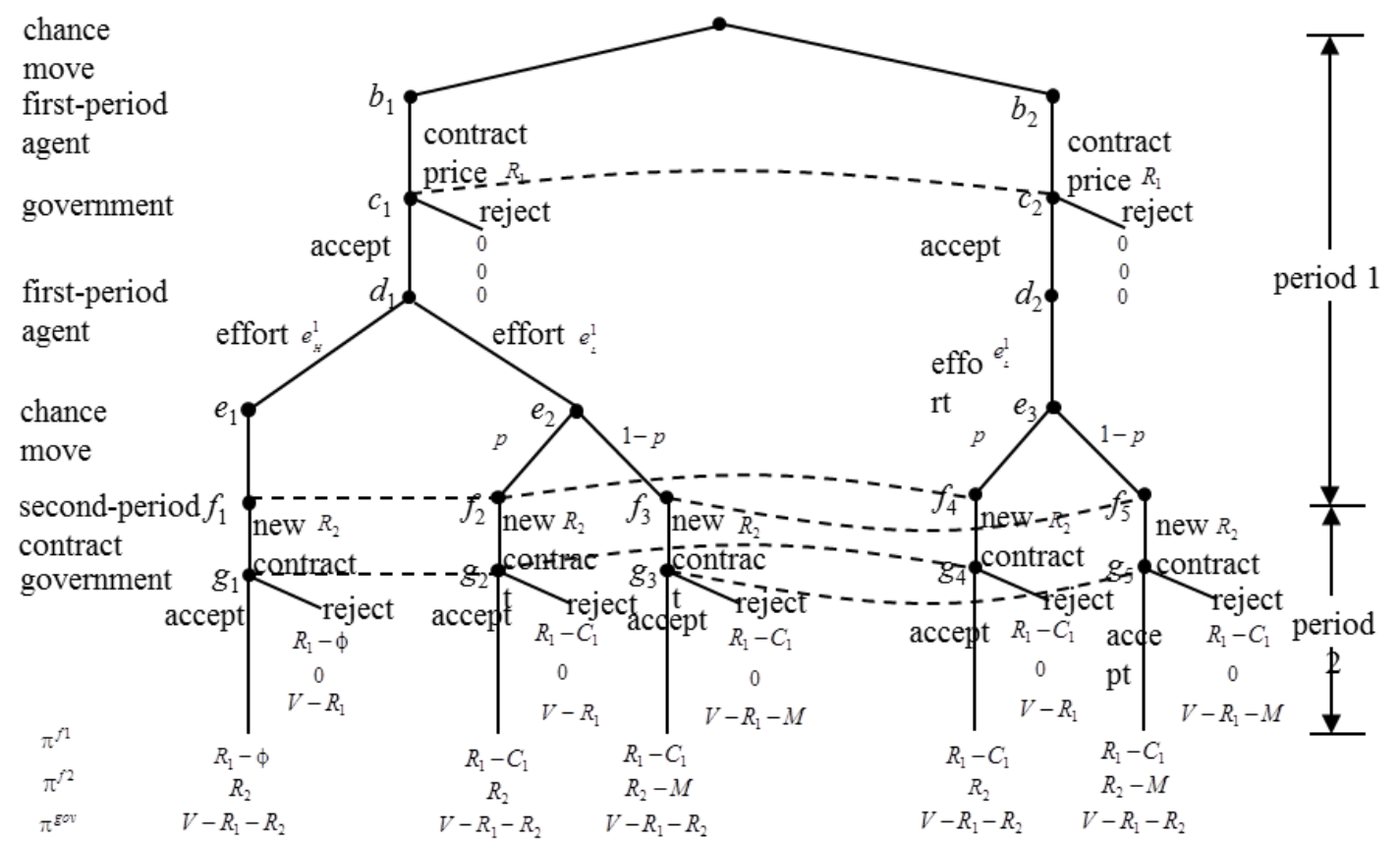

Figure 2. Game Tree of the Short-Term Contract Model

Suppose the preventative recovery cost $\phi$ satisfies

$$
\phi \geq C_{1}
$$

Assumption (3) expresses that the expected cost in the first-period when the level of effort $e_{H}^{1}$ is chosen is larger than that when the level of effort $e_{L}^{1}$ is chosen. In other words, the moral hazard problem might arise in the first period. The last assumption states that the level of effort $e_{H}^{1}$ is socially optimal.

$$
C\left(\theta_{g}^{1}, e_{H}^{1}\right)=\phi \leq C\left(\theta_{i}^{1}, e_{L}^{1}\right)=C_{1}+(1-p) M
$$

\subsection{Equilibria of Short-Term Contract}

The behaviors of relative parties that participate in the short-term contract are described as an extensive form of game, as shown in Figure 2.

In the first period, the agent chooses $e_{H}^{1}$ with $\operatorname{cost} \phi$ adopted. On the other hand, the cost related to effort is zero with ex-post recovery cost $C_{1}$ occurs if the agent chooses $e_{L}^{1}$.The agent's expected payoffs become

$$
\begin{gathered}
\pi_{\theta_{g}^{1}, H}^{f 1}=R_{1}-\phi \\
\pi_{\theta_{i}^{1}, L}^{f 1}=R_{1}-C_{1}(i=g, b)
\end{gathered}
$$

Now consider problem faced by the good agent in choosing the level of effort at the beginning of the first-period. The incentive constraint imposed on the good agent to choose $e_{H}^{1}$ is written as

$$
\pi_{\theta_{g}^{1}, H}^{f 1}-\pi_{\theta_{g}^{1}, L}^{f 1}=C_{1}-\phi \geq 0
$$

Given assumption (3), inequality (4) is not supported. As a result, the first-period agent must choose $e_{L}^{1}$. On the other hand, the condition that satisfies the agent choice of $e_{L}^{1}$ is written as 


$$
R_{1} \geq v_{1}
$$

Inequality (7) represents the participation condition for the agent who chooses $e_{L}^{1}$. Define $v_{1}=C_{1}$.

The government completes a new contract with a new agent in the second-period. The maintenance cost due to the payoff externality is dependent on the first-period agent's type and the level of effort chosen by the first-period agent. Suppose the agent chooses $e_{L}^{1}$ in the first-period. The additional cost $K$ due to the payoff externality is determined at the beginning of the second-period. The agent's expected payoffs are written as

$$
\begin{gathered}
\pi^{f 2}(0)=R_{2} \\
\pi^{f 2}(M)=R_{2}-M
\end{gathered}
$$

According to (8a) and (8b), the participation conditions are written as

$$
\begin{gathered}
R_{2} \geq 0(\text { if } K=0) \\
R_{2} \geq M(\text { if } K=M)
\end{gathered}
$$

Therefore, the equilibriums obtained under the short-term contract of the firstperiod are

Equilibrium $A$ : $\theta_{1}^{*}=\theta_{g}^{1}, e^{*}=e_{L}^{1}, R_{1} \geq v_{1}$

Equilibrium $B$ : $\theta_{1}^{*}=\theta_{b}^{1}, e^{*}=e_{L}^{1}, R_{1} \geq v_{1}$

The occurrence of Equilibrium $A$ or $B$ only depends on the agent's type. The agent's expected payoff according to the equilibriums are written as

$$
\begin{aligned}
& \text { Equilibrium } A: \pi_{\theta_{g}^{1}, H}^{f 1}=R_{1}-C_{1} \\
& \text { Equilibrium } B: \pi_{\theta_{b}^{1}, H}^{f 1}=R_{1}-C_{1}
\end{aligned}
$$

On the other hand, the second-period cost structure is determined by following the additional cost. Therefore, the second-period contract is completed under the condition (9a), (9b).

\subsection{Competitive Tender Equilibria}

Tender process at the beginning of first- and second-period is considered. The bidder that offers the minimum price will be finally selected as the contractor. The equilibriums obtained by this tender process are referred as competitive tender equilibriums. In the competitive tender, the required performance and penalty fee are disclosed in advance. Suppose that a perfect competition is accomplished during the tender process. In the real world, considerable transaction costs occur, for instance, the costs for participating in the tender, etc. Thus, the number of bidders who participate in the tender is restricted in many cases. In this sense, a perfect competition might not be accomplished. There is an extensive literature [14][15] that investigates the mechanism of the tender. As we assume that perfect competition is accomplished in the tender, the mechanism of the tender is out of the scope of this paper. 
Firstly, consider the competitive tender for the first-period contract. We find the

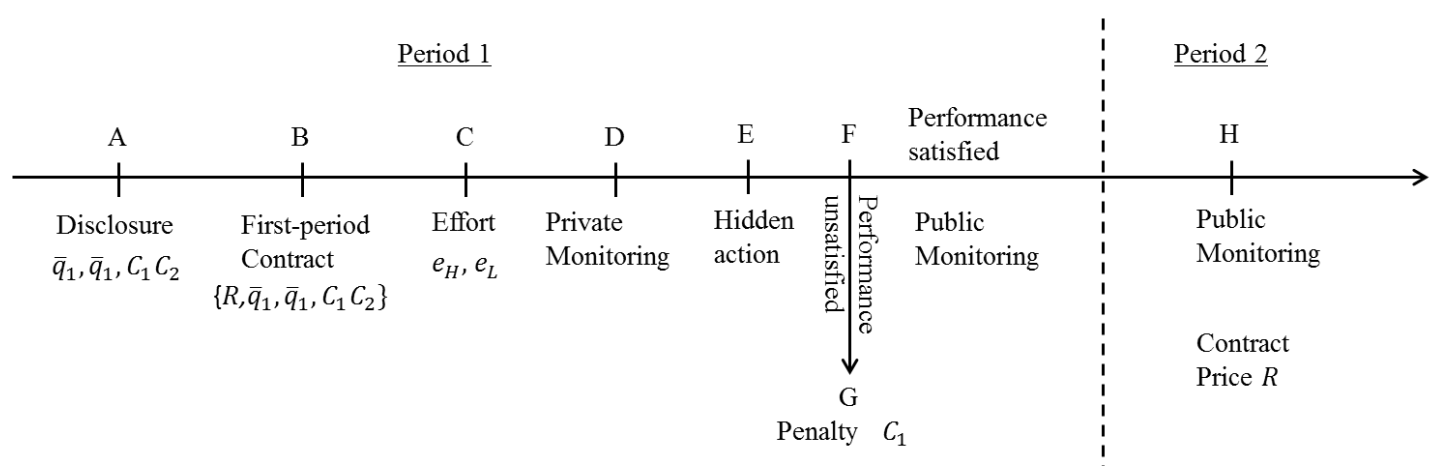

Figure 3. Timing of the Long-Term Contract

minimum price $R_{1}$ from the equilibria obtained in the short-term contract game. Denoting the minimum contract prices (hereafter marginal contract prices) in the equilibriums $\mathrm{A}$ and $\mathrm{B}$ as $R_{1 \mathrm{~min}}^{A}$ and $R_{1 \mathrm{~min}}^{B}$, respectively, it can be written as

$$
R_{1 \text { min }}^{A}=R_{1 \min }^{B}=v_{1}
$$

Thus, the competitive tender equilibrium price denoted as $R_{1}^{\circ}$ is given by

$$
R_{1}^{\circ} \geq v_{1}
$$

The competitive tender equilibria (Equilibria $S$ ) under the first-period contract are

$$
\left(\theta_{1}^{\circ}=\theta_{g}^{1}, e^{\circ}=e_{L}^{1}, R_{1}^{\circ} \geq v_{1}\right) \text { or }\left(\theta_{1}^{\circ}=\theta_{b}^{1}, e^{\circ}=e_{L}^{1}, R_{1}^{\circ} \geq v_{1}\right)
$$

In the equilibria $S$, the probability of selecting the good agent is $\alpha$ whereas the probability of selecting the bad agent $1-\alpha$. The good agent chooses the level of effort $e_{L}^{1}$ so the social optimal contract is not realized.

Now take into account a tender that determines the contract price $R_{2}$ implemented at the beginning of the second-period. The bidder that offers the minimum price $R_{2}$ is selected as the contract winner. The marginal contract price is determined accordong to the additional cost. Thus, the marginal contract prices corresponding to additional cost $K(K=0, M)$ are written as

$$
\begin{gathered}
R_{2 \min }(0)=0 \\
R_{2 \min }(M)=M
\end{gathered}
$$

According to (13a) and (13b), the competitive tender equilibriums under the equilibria $S$ are written as

$$
\begin{gathered}
R_{2}^{\circ}=0(\text { if } K=0) \\
R_{2}^{\circ}=M(\text { if } K=M)
\end{gathered}
$$

Finally, we obtain the following proposition.

Proposition 1: Under the existence of payoff externality, the adverse selection and moral hazard cannot be deterred by the competitive tender in the first-period performance-based contract. 


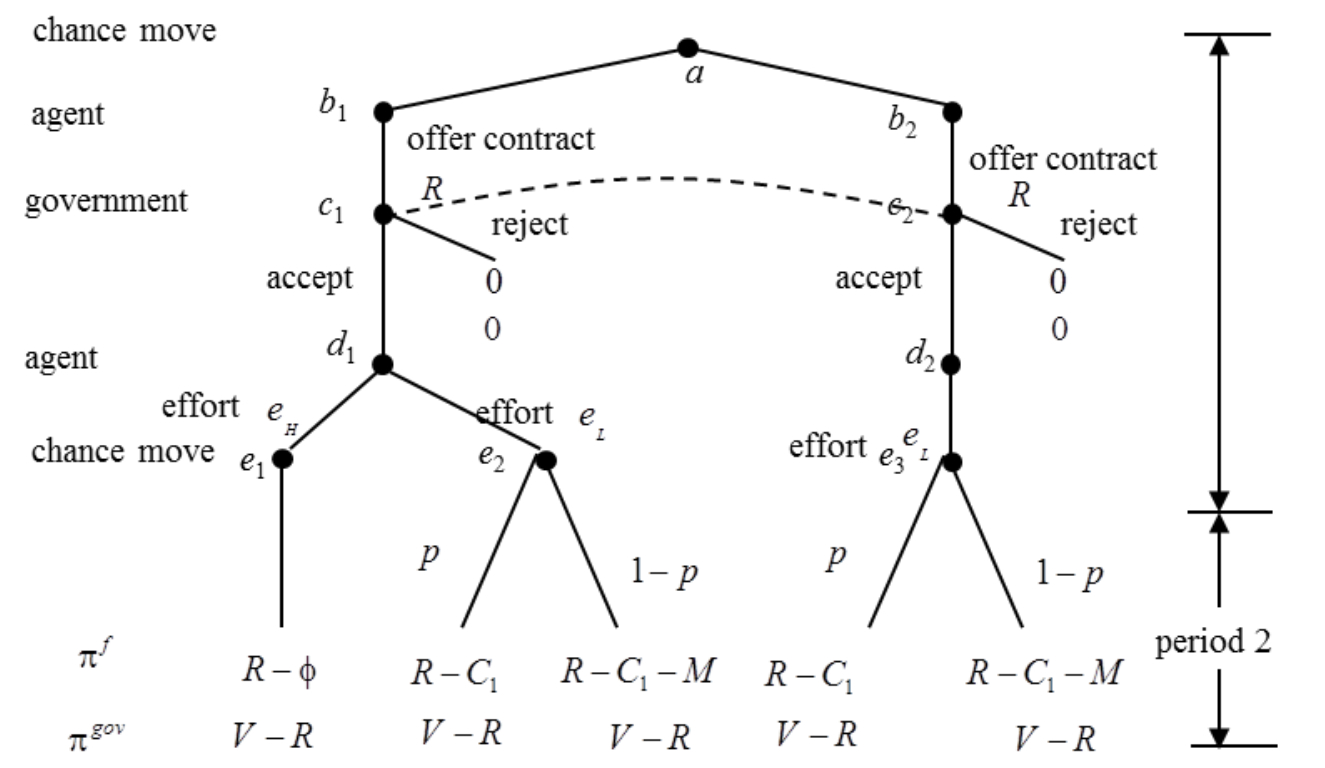

Figure 4. Game Tree of the Long-Term Contract Model

\section{Long-term Contract}

\subsection{Long-term Contract Game and Payoff Externality Internalization}

Short-term contract is incomplete because of the indescribable performance factors. Even though the describable performance can be complied by setting up the penalty clauses in the contract, the adverse selection and moral hazard cannot be deterred as described in Proposition 1 because there are no penalty clauses to keep the payoff externality away in consequence of indescribable performance factors. This section indicates the payoff externality internalized by lengthening the contract term. As a result, the moral hazard can be deterred under long-term contract. We also exclude the possibility of breaching the contract by the agent, which will be discussed in Section 4.

The long-term contract is interpreted as a 2-period game as shown in Figure 3. The cost structure is equal to that in the short-term contract; meanwhile, the payment structure is different. Suppose that the government pays the contract price in one lump sum at the end of the second-period (point H). Since the breach of the contract is forbidden, the timing of payment does not fundamentally affect the structure of the long-term contract. In fact, the outcome of long-term contract under lump sum payment will not change by devising a separate payment scheme. The government implements the monitoring at the end of the first-and second-period. The agent pays the penalty fee $C_{1}$ when the performance doesn't fit the required standard.

\subsection{Long-term Contract Game and Payoff Externality Internalization}

The behavior of relative parties participating in the short-term contract are described as an extensive form game, as shown in Figure 4. The government enters a 2-period contract with the agent. The agent's expected payoff are written as

$$
\pi_{\theta_{g}, H}=R-\phi
$$




$$
\begin{aligned}
& \pi_{\theta_{g}, L}=R-C_{1}-(1-p) M \\
& \pi_{\theta_{b}, L}=R-C_{1}-(1-p) M
\end{aligned}
$$

Now consider the problem of the good agent that faces the choice of effort level at the beginning of the first-period. The incentive constraint imposed on the agent to choose $e_{H}^{1}$ is written as

$$
\pi_{\theta_{g}, H}-\pi_{\theta_{g}, L}=C_{1}+(1-p) M-\phi \geq 0
$$

Thus, the condition for the agent choosing $e_{H}^{1}$ is written as

$$
R \geq \tau_{1}
$$

Define $\tau_{1}=\phi$. On the other hand, the bad agent chooses $e_{L}^{1}$. The bad agent's expected payoff is written as

$$
\pi_{\theta_{b}, L}=R-C_{1}+(1-p) M
$$

According to (18), the condition for the bad agent that participates in the contract is expressed by

$$
R \geq \tau_{2}
$$

Define $\tau_{2}=C_{1}+(1-p) M$. Therefore, we obtain the following equilibria of longterm contract.

$$
\begin{aligned}
& \text { Equilibrium } A^{\prime}: \theta^{*}=\theta_{g}, e^{*}=e_{H}, R \geq \tau_{1} \\
& \text { Equilibrium } B^{\prime}: \theta^{*}=\theta_{b}, e^{*}=e_{L}, R \geq \tau_{2}
\end{aligned}
$$

Note that both equilibrium $A^{\prime}$ and $B^{\prime}$ might exist if $R \geq \max \left\{\tau_{1}, \tau_{2}\right\}$. The agent's type determines which equilibrium exists. In the next section we analyze the mechanism in which the agent's type is selected by the competitive tender. The agent's expected payoffs according to the equilibria are written as

$$
\begin{aligned}
& \text { Equilibrium } A^{\prime}: \pi_{\theta_{g}, H}^{\prime}=R-\phi \\
& \text { Equilibrium } B^{\prime}: \pi_{\theta_{b}, L}^{\prime}=R-C_{1}-(1-p) M
\end{aligned}
$$

\subsection{Competitive Tender Equilibria}

A competitive tender, where the bidder offering the minimum price is selected as a long-term contractor with the government, is put into effect at the beginning of the first-period. Denote the marginal price in the equilibrium $A^{\prime}$ as $R_{\min }^{A^{\prime}}$ and write as

$$
R_{\min }^{A^{\prime}} \geq \tau_{1}
$$

Similarly, the marginal price in the equilibrium $B^{\prime}$ is given by

$$
R_{\min }^{B^{\prime}} \geq \tau_{2}
$$

Following assumption (6), $\tau_{1}<\tau_{2}$ holds. Thus the competitive price is expressed by 


$$
R^{\circ \circ}=\tau_{1}
$$

Therefore, we obtain the following competitive tender equilibrium

Competitive tender equilibrium $L$

$$
\theta^{\circ \circ}=\theta_{g}, e^{\circ \circ}=e_{H}, R^{\circ \circ}=\tau_{1}
$$

The competitive tender equilibrium $L$ means that the good agent is selected as a competitive tender, i.e., the adverse selection doesn't occur. At the same time, the agent exerts the level of effort $e_{H}$, i.e., the moral hazard is deterred, following Proposition 2 as

Proposition 2: Under the assumption that the breach of the contract is forbidden, the social optimal performance-based contract is accomplished by the long-term contract after implementing a competitive tender.

\section{Breach of long-term Contract and Efficiency}

\subsection{Uncertainty of Payoff Externality and Private Monitoring}

Previous section assumes that the breach of the long-term contract by the agent is forbidden. However, the social efficiency of the long-term contract is flawed if the agent could cancel the contract in the middle of the process. The agent might exempt from the contract if the payoff externality exists. Now consider the payoff externality that arises from the neglect of the preventative recovery, i.e., the good agent chooses the level of effort $e_{L}$, or the bad agent is selected. The agent might be intent to exempt from the contract when recognize the additional cost occurring in the second-period. As a result, the agent might escape from the additional cost due to limited liability. In this sense, the moral hazard and adverse selection might occur in the long-term contract. In this section we analyze the mechanism of moral hazard and adverse selection in the case that breach of long-term contract is possible. We furthermore investigate the deposit policy which is effective on deterring the strategic breach of the contract.

\subsection{The Long-term Contract Game Considering Breach of Contract}

Under the long-term contract that forbids the contract breach, the agent will pay the penalty fee if the real performance fails to meet the required performance. However, the agent might go bankrupt deliberately in order to avoid paying the penalty fee if contract breach is possible. As analyzed in Section 4, the execution condition that guarantees the good agent be selected is written as

$$
R \geq \tau_{1}
$$

Condition (24) is mutual information between the government and agent so in this section it is assumed that the inequality (24) holds. The contract price is paid by the government at the end of the second-period. In other words, the agent cannot benefit from the payoff unless completes the contract. In spite of this strong assumption, in this section the possibility of the contract violation is indicated.

Firstly, consider the case in which the real performance at the end of the firstperiod fails to meet the level of required performance. The agent obtains the information about the additional cost occurring in the second-period through the private monitoring. At this moment, the information is private since the public 
monitoring is not implemented yet. Now consider the case that the additional cost is identified as 0 . The payoff of the agent when the contract continues is written as

$$
\tilde{\pi}_{c}(0)=R-C_{1} .
$$

On the other hand, the agent's payoff when the contract ends is written as

$$
\tilde{\pi}_{q}(0)=0 .
$$

Thus, the condition that satisfy the agent continue the contract is

$$
\tilde{\pi}_{c}(0)-\tilde{\pi}_{q}(0)=R-C_{1} \geq 0
$$

According to assumption (3) and (24), inequality (27) must hold. As a result, the agent must choose to continue the contract when the additional cost is identified as 0 .

Then consider the case that the additional cost is identified as $M$. The agent earns the following payoff when the contract continues.

$$
\tilde{\pi}_{c}(M)=R-C_{1}-M
$$

However, the agent's payoff when the contract ends is written as

$$
\tilde{\pi}_{q}(M)=0
$$

Therefore, the continuation condition is expressed by

$$
\tilde{\pi}_{c}(M)-\tilde{\pi}_{q}(M)=R-C_{1}-M \geq 0
$$

On the contrary, the agent will withdraw from the maintenance works by quitting the contract if condition (30) does not hold.

\subsection{Equilibria and Competitive Tender Equilibria}

In the long-term contract the breach of contract is considered, the equilibria are determined according to the condition (30).

Case a): $R \geq C_{1}+M$

In this case, $R \geq C_{1}+M$ is assumed. Given (30), the agent chooses to stay. As discussed in Section 4, the good agent must exert effort $e_{H}$. According to (17), the conditions for the contract are expressed by

$$
\begin{aligned}
& R \geq \tau_{1} \\
& R \geq \tilde{\tau}_{1}
\end{aligned}
$$

Define $\tilde{\tau}_{1}=C_{1}+M$. Inequality (31a) represents the incentive condition for the level of effort $e_{H}$ be chosen, whereas inequality (31b) represents the agent's participation condition. The condition (31b) holds as a result of the assumption in this case. Given assumption (3), $\tilde{\tau}_{1}>\tau_{1}$. Therefore, the implementation condition with respect to the level of effort $e_{H}$ is written as

$$
R \geq \tilde{\tau}_{1}
$$

On the other hand, the establishing conditions when the bad agent is selected are written as

$$
R \geq \tau_{2}
$$




$$
R \geq \tilde{\tau}_{1}
$$

Inequality (33a) represents the bad agent's participation condition, whereas (33b) represents the establishing condition of this case. Noting $\tilde{\tau}_{1}>\tau_{2}$, the condition for the bad agent participating in the contract is written as

$$
R \geq \tilde{\tau}_{1}
$$

Therefore we obtain the following equilibria

$$
\begin{aligned}
& \text { Equilibrium } \tilde{A}: \tilde{\theta}^{*}=\theta_{g}, \tilde{e}^{*}=e_{H}, R \geq \tilde{\tau}_{1} \\
& \text { Equilibrium } \tilde{B}: \tilde{\theta}^{*}=\theta_{b}, \tilde{e}^{*}=e_{L}, R \geq \tilde{\tau}_{1}
\end{aligned}
$$

Both equilibrium $\tilde{A}$ and $\tilde{B}$ might be feasible and the result depends on the agent's type determined before the contract is completed.

Case b): $\phi \leq R<C_{1}+M$

In this case, the real performance at the end of the first-period doesn't satisfy the required performance, the ex-post recovery cost $C_{1}$ is obligated subsequently. The agent then chooses to continue the contract after identifying the additional cost in the second-period will be zero. However, the agent quits the contract when the additional cost is $M$. According to assumption (1), the real performance of the firstperiod will meet the required performance if the agent choses the level of effort $e_{H}$. Afterwards no additional cost occurs. Therefore, the contact violation doesn't exist if the agent chooses $e_{H}$. Given (15a), the agent's payoff is expressed by

$$
\tilde{\pi}_{\theta_{g}, H}=R-\phi
$$

On the other hand, the agent earns payoffs when choosing $e_{L}$ are written as

$$
\begin{aligned}
& \tilde{\pi}_{\theta_{g}, L}=p R, \\
& \tilde{\pi}_{\theta_{b}, L}=p R
\end{aligned}
$$

The conditions ensuring the good agent to choose the level of effort $e_{H}$ are expressed by

$$
\begin{gathered}
\tilde{\pi}_{\theta_{g}, H}-\tilde{\pi}_{\theta_{g}, L}=R-\phi-p R \geq 0, \\
R \geq \tau_{1} \\
R<\tilde{\tau}_{1}
\end{gathered}
$$

Inequality (37a) represents the incentive constraint imposed on the agent to choose $e_{H}$, whereas (37b) represents the implementation condition with respect the level of $e_{H}$. (37c) corresponds to the assumption of this case. Given (37a), we obtain the following inequality.

$$
R \geq \tilde{\tau}_{2}
$$

Define $\tilde{\tau}_{2}=\phi /(1-p)$. Given $\tilde{\tau}_{2}>\tau_{1}=\phi$, the implementation condition with respect to the level of effort $e_{H}$ is written as 


$$
\tilde{\tau}_{1}>R \geq \tilde{\tau}_{2}
$$

On the other hand, the good agent chooses the level of effort $e_{L}$ if the condition (37a) doesn't hold. Therefore, the conditions with respect to $e_{L}$ are written as

$$
\begin{gathered}
R<\tilde{\tau}_{2} \\
R \geq 0 \\
\tau_{1} \leq R<\tilde{\tau}_{1}
\end{gathered}
$$

Inequality (40a) represents the incentive constraint imposed on the agent to choose $e_{L}$, whereas (40b) represents the participation condition with respect the level of $e_{L}$. (40c) corresponds to the assumption of this case. Note (40c) ensures that the participation condition (40b) is satisfied. The implementation condition with respect to $e_{L}$ is subsequently written as

$$
\min \left\{\tilde{\tau}_{1}, \tilde{\tau}_{2}\right\}>R \geq \tau_{1}
$$

On the other hand, the implementation conditions with respect to the bad agent are expressed by

$$
\begin{aligned}
& R \geq 0 \\
& R \geq \tau_{1}
\end{aligned}
$$

Inequality (42a) indicates the participation condition, whereas (42b) corresponds to the assumption of this case. Note (42b) ensures that (42a) holds. The implementation condition with respect to the bad agent is thus written as

$$
R \geq \tau_{1}
$$

Therefore, the following equilibria are obtained

$$
\begin{aligned}
& \text { Equilibrium } \tilde{C}: \tilde{\theta}^{*}=\theta_{g}, \tilde{e}^{*}=e_{H}, \tilde{\tau}_{1}>R \geq \tilde{\tau}_{2} \\
& \text { Equilibrium } \widetilde{D}: \tilde{\theta}^{*}=\theta_{g}, \tilde{e}^{*}=e_{L}, \min \left\{\tilde{\tau}_{1}, \tilde{\tau}_{2}\right\}>R \geq \tau_{1} \\
& \text { Equilibrium } \tilde{E}: \tilde{\theta}^{*}=\theta_{b}, \tilde{e}^{*}=e_{L}, \tilde{\tau}_{1}>R \geq \tau_{1}
\end{aligned}
$$

The moral hazard or adverse selection occurs in above equilibriums of the longterm contract considering the breach of contract.

Next consider that the bidder offering the minimum contract price is selected as the winning bidder by a competitive tender. The bidders who attend the tender might be good agent or bad agent. Denote the marginal prices in the equilibriums $\tilde{A}$ and $\tilde{B}$ by $R_{\min }^{\tilde{A}}$ and $R_{\min }^{\tilde{B}}$ respectively, which are written as

$$
R_{\min }^{\tilde{A}}=R_{\min }^{\tilde{B}}=\tilde{\tau}_{1}
$$

The marginal prices in the equilibria $\tilde{C}, \widetilde{D}$ and $\widetilde{E}$ are denoted by $R_{\min }^{\tilde{C}}, R_{\min }^{\widetilde{D}}$ and $R_{\text {min }}^{\tilde{E}}$ written as

$$
\tilde{\tau}_{1}>R_{\min }^{\tilde{C}}=\tilde{\tau}_{2}
$$




$$
\begin{gathered}
\min \left\{\tilde{\tau}_{1}, \tilde{\tau}_{2}\right\}>R_{\min }^{\widetilde{\widetilde{D}}}=\tau_{1} \\
\tilde{\tau}_{1}>R_{\min }^{\tilde{E}}=\tau_{1}
\end{gathered}
$$

Given the definition of $\tilde{\tau}_{1}, \tilde{\tau}_{2}$ and $\tau_{1}$, the following order relations of the marginal price are obtained

$$
\begin{aligned}
& \text { Pattern 1: } \tilde{\tau}_{1} \geq \tilde{\tau}_{2}>\tau_{1} \\
& \text { Pattern 2: } \tilde{\tau}_{2} \geq \tilde{\tau}_{1}>\tau_{1}
\end{aligned}
$$

Because $\min \left\{\tilde{\tau}_{1}, \tilde{\tau}_{2}\right\}>\tau_{1}$ is satisfied in both patterns, the competitive tender price is determined as

$$
\tilde{R}^{\circ \circ}=\tau_{1}
$$

Therefore, we obtain the following equilibriums

\section{The competitive tender equilibria $\tilde{L}$}

$$
\begin{aligned}
& \left(\tilde{\theta}^{\circ \circ}=\theta_{g}, \tilde{e}^{\circ \circ}=e_{L}, \tilde{R}^{\circ \circ}=\tau_{1}\right) \text { or } \\
& \left(\tilde{\theta}^{\circ \circ}=\theta_{b}, \tilde{e}^{*}=e_{L}, \tilde{R}^{\circ \circ}=\tau_{1}\right)
\end{aligned}
$$

In the equilibria $\tilde{L}$, the good agent and bad agent are selected with probability $\alpha$ and $1-\alpha$ respectively. However, the socially optimal contract is not accomplished as the level of effort $e_{L}$ is chosen by the agent who is good or bad. Furthermore, the agent quits the contract when finding the additional cost occurring in the secondperiod is $M$ through the private monitoring. In other words, the moral hazard and adverse selection cannot be deterred if the agent could breach the long-term contract. The result is summarized as the following proposition.

Proposition 3: The moral hazard and adverse selection occur if the breach of contract cannot be deterred under the long-term contract.

\subsection{Deposit Game}

Now consider the following scheme called deposit policy. Suppose the government imposes an amount of deposit from the agent at the beginning of the first-period. When the agent quits the contract during the contract period, the imposed deposit is confiscated by the government. The confiscated deposit then is used to the recovery of the asset. On the other hand, the imposed deposit is returned to the agent when the agent continues the project until period 2. At the point in time when the agent identifies the additional cost $M$ occurring through the private monitoring, the condition ensuring the agent to continue the contract is written as

$$
R \geq C_{1}+M-E
$$

Now suppose inequality (48) is satisfied. Given (48), the agent continues the contract until the second-period even though finding the additional cost $M$ occurring. In this sense, we obtain the same equilibrium in the deposit game as in the long-term game as well as the competitive tender equilibrium, which is expressed by 
The competitive tender equilibrium $\hat{L}$

$$
\left(\hat{\theta}^{\circ \circ}=\theta_{g}, \hat{e}^{\circ \circ}=e_{H}, \hat{R}^{\circ \circ}=\tau_{1}\right)
$$

That is, the good agent who exerts the level of effort $e_{H}$ is selected under the condition of $R=\tau_{1}=\phi$. Given (48), the optimal deposit is determined as

$$
E^{*} \geq C_{1}+M-\phi
$$

Given (49), the good agent is selected, i.e., the adverse selection is avoided. Furthermore, the selected agent chooses the level of effort $e_{H}$, i.e., the moral hazard is deterred. We subsequently obtain the following proposition.

Proposition 4: When the government imposes the deposit satisfying (49) from the agent at the beginning of the long-term contract, both the adverse selection and moral hazard can be deterred.

\subsection{Discussion}

As it is shown in Proposition 1, the adverse selection and moral hazard cannot be deterred under the short-term contract. Proposition 2 indicates the long-term contract is optimal under the assumption that the breach of contract is forbidden. The above propositions suggest the advantage of the long-term contract. However, adverse selection due to low priced bidding and moral hazard arise under the longterm contract if the agent is not obligated to commit the contract. In other words, the ensuring of the long-term contract efficiency depends on the commitment of completing the contract by the agent.

Proposition 4 theoretically indicates that the optimal long-term contract is accomplished by adopting the deposit policy. However, the introduction of deposit is open to further discussion. The fact that deposit is imposed for the commitment of completing the contract instead of penalty fee is not to be argued. However, inefficient opportunity cost with respect to the imposed deposit exists. In order to restrain the opportunity cost beard by the agent, the government needs to effectively operate the deposit. In this sense, the design of the institutional framework for the effective operation of the deposit is necessary. On the other hand, the project risks increase along with the amount of deposit becomes larger. As a result, the number of the agents attending the project becomes decreased.

From good agent's perspective, the deposit doesn't need to be beard if the adverse selection can be resolved. In the real world, obtaining the good agents' consensus about deposit is not easy issue. Instead enhancement of nominated competitive tender based on the rigorous screening on the technological ability becomes important. Needless to say, the good agent might breach the contract. However, the maintenance works of the infrastructure assets are characterized by the long-term and continuity. Under the long-term contract with strong continuity, the reputation mechanism is considered to be possible to deter the breach of contract by the agent. Granted that the deposit is introduced, the technological screening should be implemented in order to decrease the amount of deposit.

\section{Conclusion}

In this paper, we attempted to investigate the structure of the performance-based contract for the management and operation works of the transport infrastructure. In the performance-based contract, the adverse selection and moral hazard might arise from the payoff externality and the private monitoring implemented by the agent. We analyzed the effect of the contract period, penalty clauses and the competitive 
tender upon the adverse selection and moral hazard, whereby the main focus is on that the payoff externality is internalized in the long-term contract. The following conclusions are obtained. (1) The adverse selection and moral hazard cannot be deterred by the penalty system and competitive tender if the maintenance works are carried out by short-term contract. (2) The adverse selection and moral hazard can be efficiently deterred by the long-term contract, as far as the agent is forbidden to quit from the contract throughout the contract periods. (3) The deposit is effective to deter the inefficiency arises from the breach of contract. The issues above hold under assumptions in this paper. A discussion about the efficiency of the performance-based contract from different approach is necessary. Firstly, this paper supposes the asset is renewed after the life period terminates, which ensures the payoff externality is internalized in the long-term contract. However, in the real world the renewal might not be implemented soon after the life period finished. This situation needs alternative means to deter the agent's strategic behavior instead of lengthening the contract period. Secondly, the theoretical study about the device for deterring the breach of contract should be accumulated. The institutional design for decreasing the opportunity cost of the deposit is necessary when considering adopting the deposit policy. Thirdly, the payment structure of contract price should be more investigated. In this paper, we suppose the payment takes place at the ending of each period. This assumption emphasizes that the agent still might quit from the contract even though it gives the agent the strongest incentive to commit the contract. In the real world, the contract price is paid each fiscal year. The desirable payment structure easing the risks beard by the agent, as well as ensuring the implementation of the optimal performance-based contract should be developed in the future.

Tendency

\section{References}

[1] G. F. Segal, A. T. Moore and S. McCarthy, Contracting for Road and Highway Maintenance, Reason Public Policy Institute (2003).

[2] P. Bolton and M. Dewatripont, Contract Theory, The MIT Press (2005).

[3] B. Salanié, The Economics of Contracts, The MIT Press (1997).

[4] D. P. Baron, and D. Besanko, Regulation, information in a continuing relationship, Information Economics and Policy, 1, 447-470 (1984).

[5] X. Freixas, R. Guesnerie and J. Tirole, Planning under incomplete information and the Ratchet effect, Review of Economic Studies, 52, 173-192 (1985).

[6] J. J. Laffont and J. Tirole, "A Theory of Incentives in Procurement and Regulation", The MIT Press (1993).

[7] J. J. Laffont and J. Tirole, "Adverse selection and renegotiation in procurement", Review of Economic Studies, vol. 57, (1991), pp. 597-625.

[8] W. P. Rogerson, "Repeated moral hazard", Econometrica, vol. 53, (1985), pp. 69-76.

[9] D. Fudenberg, B. Holmström and P. Milgrom, "Short-term contracts and long-term agency relationships", Journal of Economic Theory, vol. 51, (1990), pp. 1-31.

[10] P.-A. Chiappori, I. Macho, P. Rey and B. Salanié, "Repeated moral hazard: The role of memory, commitment, and the access to credit markets", European Economic Review, vol. 38, (1994), pp. 15271553.

[11] P. W. Schmitz, "Allocating control in agency problems with limited liability and sequential hidden actions", Rand Journal of Economics, vol. 36, (2005), pp. 318-336.

[12] T. Sekiguchi, "Efficiency in repeated prisoner's dilemma with private monitoring", Journal of Economic Theory, vol. 76, (1997), pp. 345-361.

[13] J. C. Stein, "Takeover threats and managerial myopia”, Journal of Political Economy, vol. 96, (1988), pp. 61-81.

[14] E. L. Von Thadden, "Long-term contracts, short-term investment and monitoring", Review of Economic Studies, vol. 62, (1995), pp. 557-575.

[15] P. Klemperer, "Auctions: Theory and Practice", Princeton University Press, (2003).

[16] P. Milgrom, "Putting Auction Theory to Work", Cambridge University Press, (2004). 


\section{Authors}

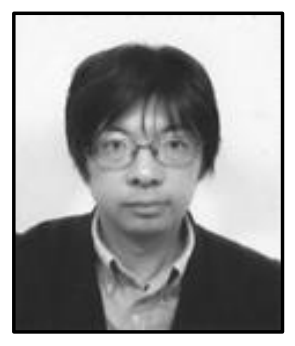

Lei Shi, BSc., MSc., Dr. Eng., is associate professor at Department of Construction Management, Dalian University of Technology, Dalian, China. Dr. Shi earned the doctoral degree in Urban Management from Kyoto University (Japan) and master degree in Architecture and Building Science from Tohoku University (Japan); as well as the Bachelor's degree in Architecture from Dalian University of Technology. His research interests include Construction Contract, Construction Institutional Design, Infrastructure Management and Risk Management.

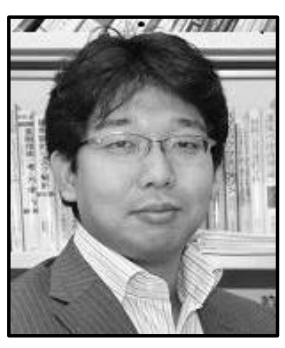

Masamitsu Onishi, BSc., MSc., Dr Eng., is Assistant Professor at the Department of Urban Management, Graduate School of Engineering, Kyoto University, Japan. His doctoral degree was awarded by Kyoto University. He has published 40 academic papers which concerns dispute resolution in construction projects, public private partnership, project financing. Dr. Onishi is a board member of Research Committee of Private Finance Initiative in Japan Society of Civil Engineering (JSCE). He is a recipient of JSCE young researcher award 2006 and best paper award 2008.

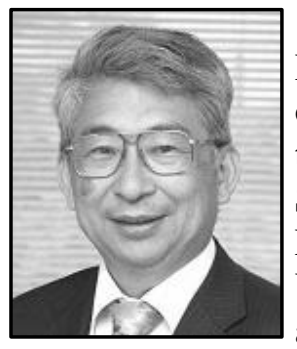

Kiyoshi Kobayashi, BSc., MSc., Dr Eng., Kyoto University, is a Professor of infrastructure economics at Kyoto University. He serves on the editorial boards of the American Society of Civil Engineers, the Annals of Regional Science, the Journal of Applied Regional Science and the Japan Journal of Civil Engineering. He was also the Editor in chief for the Journal of Japan Society of Civil Engineers and Vice Editor in chief, Journal of Infrastructure Systems. He is the author and co-editor of 53 books and more than 370 technical papers. 